\title{
SUBORDINATION FAMILIES AND EXTREME POINTS
}

\author{
YUSUF ABU-MUHANNA AND D. J. HALLENBECK
}

\begin{abstract}
Let $s(F)$ denote the set of functions subordinate to a univalent function $F$ in $\Delta$ the unit disk. Let $B_{0}$ denote the set of functions $\phi(z)$ analytic in $\Delta$ satisfying $|\phi(z)|<1$ and $\phi(0)=0$. We prove that if $f=F \circ \phi$ is an extreme point of $s(F)$, then $\phi$ is an extreme point of $B_{0}$. Let $D=F(s)$ and $\lambda(w, \partial D)$ denote the distance between $w$ and $\partial D$ (boundary of $D$ ). We also prove that if $\phi$ is an extreme point of $B_{0}$ and $\left|\phi\left(e^{i t}\right)\right|<1$ for almost all $t$, then $\int_{0}^{2 \pi} \log \lambda\left(F\left(\phi\left(e^{i t}\right) e^{i \theta}\right), \partial D\right) d t=-\infty$ for almost all $\theta$.
\end{abstract}

Introduction. Let $\Delta=\{z:|z|<1\}$ and let $\mathbf{A}$ denote the set of functions analytic in $\Delta$. Let $B_{0}$ denote the subset of $\mathbf{A}$ consisting of all functions $\phi$ that satisfy the conditions $|\phi(z)|<1, \phi(0)=0$. Let $E B_{0}$ denote the extreme points of $B_{0}$. Let $S$ denote the subset of $\mathbf{A}$ consisting of univalent functions $f$ so that $f(z)=z+\cdots$ in $\Delta$.

Let $F$ be in $\mathbf{A}$ and be univalent in $\Delta$. Let $s(F)$ denote the subset of $\mathbf{A}$ consisting of functions $f$ that are subordinate to $F$ in $\Delta$. This means that $f \in \mathbf{A}, f(0)=F(0)$, and $f(\Delta) \subset F(\Delta)$. These conditions are equivalent to the existence of $\phi \in B_{0}$ so that $f=F \circ \phi$. Note that $s(F)=\left\{F \circ \phi: \phi \in B_{0}\right\}$.

Let $D$ denote $F(\Delta)$. It is known that $F \in H^{p}$ for all $p<1 / 2[3$, p. 50] and so if $f=F \circ \phi$ for $\phi \in B_{0}$, then $f \in H^{p}$ for $p<1 / 2$ [3, pp. 10-11]. It follows that $\lim _{r \rightarrow 1} f\left(r e^{i \theta}\right)=f\left(e^{i \theta}\right)$ exists almost everywhere.

We let $E s(F)$ denote the set of extreme points of $s(F)$. In [1] it was proved that if $F^{\prime}$ is in the Nevanlinna class and $D$ is a Jordan domain subset to a half plane, then $E s(F) \subset\left\{F \circ \phi: \phi \in E B_{0}\right\}$. In that paper it was conjectured that this inclusion is valid for any univalent $F$. In [4] the inclusion was proved under the assumption $F$ is univalent and $D$ is a Jordan domain. In this paper we prove the inclusion for an arbitrary univalent function and so verify the conjecture made by the first author in $[\mathbf{1}]$.

In [1] it was also proved that if $F^{\prime}$ is in the Nevanlinna class and $\phi \in E B_{0}$, then $\int_{0}^{2 \pi} \log \lambda\left(F\left(\phi\left(e^{i t}\right)\right), \partial D\right) d t=-\infty$. It was conjectured that the integral was $-\infty$ for any univalent function $F$ when $\phi \in E B_{0}$. (Note that this is trivially true if $\left|\phi\left(e^{i t}\right)\right|=1$ on a set of positive measure since $F$ is univalent.) A weaker conjecture is that if $F \circ \phi \in E s(F)$ and $F$ is univalent, then $\int_{0}^{2 \pi} \log \lambda\left(F\left(\phi\left(e^{i t}\right)\right), \partial D\right) d t=-\infty$.

In this paper we show that this weaker conjecture holds when $F^{\prime}$ is in the Nevanlinna class [3, p. 16]. We also take this opportunity to point out that in the proof

Received by the editors Octrober 29, 1986.

1980 Mathematics Subject Classification (1985 Revision). Primary 30C80; Secondary 30C55.

Key words and phrases. Extreme point, Nevanlinna class, subordination, univalent function.

The research of the first author was completed while on sabbatical leave from the University off Petroleum and Minerals, Dhahran, Saudi Arabia. 
of Theorem 8.25 in [5, pp. 143-144] there is a proof of this weaker conjecture when $F(\Delta)$ is a Jordan domain and no assumption on $F^{\prime}$ is made. Also it is known that $\int_{0}^{2 \pi} \log \lambda\left(f\left(\phi\left(e^{i t}\right)\right), \partial D\right) d t=-\infty$ does not in general imply $F \circ \phi \in E s(F)$. This can be easily seen by considering the case $F(z)=((1+z) /(1-z))^{\alpha}$ for $0<\alpha<1[\mathbf{5}$, pp. 131, 133]. We prove the interesting result that if $F$ is univalent and $\phi \in E B_{0}$, then $\int_{0}^{2 \pi} \log \lambda\left(F\left(\phi\left(e^{i t}\right) e^{i \theta}\right), \partial D\right) d t=-\infty$ for almost all $\theta$.

\section{Functions subordinate to a univalent function.}

THEOREM 1. If $F$ is a univalent function analytic in $\Delta$ and $f=F \circ \phi$ is an extreme point of $s(F)$, then $\phi$ is an extreme point of $B_{0}$.

Proof. Assume $f=F \circ \phi \in E s(F)$ and $\phi \notin E B_{0}$. Then $\int_{0}^{2 \pi} \log \left(1-\left|\phi\left(e^{i t}\right)\right|\right) d t>$ $-\infty\left[3\right.$, p. 125]. Clearly $\left|\phi\left(e^{i t}\right)\right|<1$ almost everywhere and $\log \left(1-\left|\phi\left(e^{i t}\right)\right|\right) \in L^{1}$. Let $P_{z}(t)$ for $|z|<1$ denote $\operatorname{Re}\left(\left(e^{i t}+z\right) /\left(e^{i t}-z\right)\right)$. Since $\phi \in H^{1}$ we have $\phi\left(e^{i t}\right) \in L^{1}$ and for $|z|<1, \phi(z)=(1 / 2 \pi) \int_{0}^{2 \pi} P_{z}(t) \phi\left(e^{i t}\right) d t[\mathbf{3}$, p. 34]. It follows that

$$
1-|\phi(z)| \geq \frac{1}{2 \pi} \int_{0}^{2 \pi} P_{z}(t)\left(1-\left|\phi\left(e^{i t}\right)\right|\right) d t
$$

Applying Jensen's inequality we obtain from (1)

$$
\log (1-|\phi(z)|) \geq \frac{1}{2 \pi} \int_{0}^{2 \pi} P_{z}(t) \log \left(1-\left|\phi\left(e^{i t}\right)\right|\right) d t .
$$

Hence we deduce from (2) that

$$
\exp \left(\frac{1}{2 \pi} \int_{0}^{2 \pi} P_{z}(t) \log \left(1-\left|\phi\left(e^{i t}\right)\right|\right)^{2} d t\right) \leq(1-|\phi(z)|)^{2}
$$

Now define

$$
g(z)=\frac{z}{16} \exp \frac{1}{2 \pi} \int_{0}^{2 \pi} \frac{e^{i t}+z}{e^{i t}-z} \log \left(1-\left|\phi\left(e^{i t}\right)\right|\right)^{2} d t .
$$

Note that $g(0)=0, g \not \equiv 0$, and $g$ is analytic in $\Delta$. We also have

$$
|g(z)|<\frac{1}{16} \exp \left(\frac{1}{2 \pi} \int_{0}^{2 \pi} P_{z}(t) \log \left(1-\left|\phi\left(e^{i t}\right)\right|\right)^{2} d t\right)
$$

and so this fact and (3) imply

$$
|g(z)|<\frac{1}{16}(1-|\phi(z)|)^{2}
$$

Since $F$ is univalent, it follows from [7, p. 22] that

$$
\frac{1}{4}\left(1-|z|^{2}\right)\left|F^{\prime}(z)\right| \leq \lambda(F(z), \partial D) \leq\left(1-|z|^{2}\right)\left|F^{\prime}(z)\right|
$$

for all $z$ in $\Delta$. We may assume, without loss of generality, that $F \in S$ and so it is known that

$$
\frac{1-|z|}{(1+|z|)^{3}} \leq\left|F^{\prime}(z)\right|
$$

for all $z$ in $\Delta[7$, p. 21]. It follows from (5) and (6) that

$$
\frac{1}{16}(1-|z|)^{2} \leq \lambda(F(z), \partial D) \text {. }
$$


Therefore $\frac{1}{16}(1-|\phi(z)|)^{2} \leq \lambda(F(\phi(z)), \partial D)=\lambda(f(z), \partial D)$. Hence, by (4) and (7) we have $|g(z)|<\lambda(f(z), \partial D)$ for all $z$ in $\Delta$. We conclude that $f(z) \pm g(z) \in F(\Delta)$ for all $z$ in $\Delta$. Since $F$ is univalent, $f(0)=F(0)$, and $g(0)=0$, we have $f \pm g \in s(F)$ and $g \equiv 0$. This contradicts the assumption that $f \in E s(F)$.

Hence $\phi \in E B_{0}$ and the proof is complete.

REMARK. The conjecture made by the first author in [1] is now proved.

THEOREM 2. If $F$ is a univalent function analytic in $\Delta, f=F \circ \phi \in E s(F)$, and $F^{\prime}$ is in the Nevanlinna class, then

$$
\int_{0}^{2 \pi} \log \lambda\left(F\left(\phi\left(e^{i t}\right)\right), \partial D\right) d t=-\infty
$$

PROOF. Theorem 1 implies $F \circ \phi \in E B_{0}$ and so we have $\int_{0}^{2 \pi} \log \left(1-\left|\phi\left(e^{i t}\right)\right|\right) d t=$ $-\infty\left[3\right.$, p. 125]. It follows from this fact and the assumption that $F^{\prime}$ is in the Nevanlinna class that (8) holds [1, p. 440].

REMARK. This result was previously known under the assumptions that $F(\Delta)$ is a Jordan domain and $\phi \in E B_{0}[5$, pp. 143-144]. We conjecture that (8) holds in general. The following theorem is indirect evidence for this conjecture. then

THEOREM 3. If $F$ is a univalent function analytic in $\Delta$ and $f=F \circ \phi \in E s(F)$,

$$
\inf _{r} \int_{0}^{2 \pi} \log \lambda\left(F\left(r e^{i t}\right), \partial D\right) d t=-\infty .
$$

PROOF. We first prove that if $\phi \in B_{0}$, then

$$
\inf _{r} \int_{0}^{2 \pi} \log \left(1-\left|\phi\left(r e^{i t}\right)\right|^{2}\right) d t=\int_{0}^{2 \pi} \log \left(1-\left|\phi\left(e^{i t}\right)\right|^{2}\right) d t .
$$

It is easy to verify by a computation that $-\log \left(1-|z|^{2}\right)$ is a $C^{\infty}$ subharmonic function in $\Delta$. It follows easily by another computation that $-\log \left(1-|\phi(z)|^{2}\right)$ is subharmonic in $\Delta$ and so $-\int_{0}^{2 \pi} \log \left(1-\left|\phi\left(r e^{i t}\right)\right|^{2}\right) d t$ is an increasing function of $r$ [3, p. 9].

First consider the case $\int_{0}^{2 \pi} \log \left(1-\left|\phi\left(e^{i t}\right)\right|^{2}\right) d t=-\infty$. Then by Fatou's lemma we have

$$
+\infty=-\int_{0}^{2 \pi} \log \left(1-\left|\phi\left(e^{i t}\right)\right|^{2}\right) d t \leq \underline{\lim _{r \rightarrow 1}}-\int_{0}^{2 \pi} \log \left(1-\left|\phi\left(r e^{i t}\right)\right|^{2}\right) d t .
$$

Since $-\int_{0}^{2 \pi} \log \left(1-\left|\phi\left(r e^{i t}\right)\right|^{2}\right) d t$ is an increasing function of $r$, we have

$$
\varliminf_{r \rightarrow 1}-\int_{0}^{2 \pi} \log \left(1-\left|\phi\left(r e^{i t}\right)\right|^{2}\right) d t=\sup _{r}-\int_{0}^{2 \pi} \log \left(1-\left|\phi\left(r e^{i t}\right)\right|^{2}\right) d t=+\infty .
$$

It follows that inf $r \int_{0}^{2 \pi} \log \left(1-\left|\phi\left(r e^{i t}\right)\right|^{2}\right) d t=-\infty$ and (10) holds in this case.

Now suppose $\int_{0}^{2 \pi} \log \left(1-\left|\phi\left(e^{i t}\right)\right|^{2}\right) d t>-\infty$. We have $\log \left(1-\left|\phi\left(e^{i t}\right)\right|^{2}\right) \in L^{1}$, and it follows by the type of arguments used in the proof of Theorem 1 that

$$
-\log \left(1-\left|\phi\left(r e^{i \theta}\right)\right|^{2}\right) \leq \frac{-1}{2 \pi} \int_{0}^{2 \pi} P_{z}(t) \log \left(1-\left|\phi\left(e^{i t}\right)\right|^{2}\right) d t
$$


Hence by integrating both sides of (12), using Fubini's theorern and the fact that $(1 / 2 \pi) \int_{0}^{2 \pi} P_{z}(t) d \theta=1$ we have

$$
-\int_{0}^{2 \pi} \log \left(1-\left|\phi\left(r e^{i t}\right)\right|^{2}\right) d t \leq-\int_{0}^{2 \pi} \log \left(1-\left|\phi\left(e^{i t}\right)\right|^{2}\right) d t .
$$

It follows that

$$
\int_{0}^{2 \pi} \log \left(1-\left|\phi\left(e^{i t}\right)\right|^{2}\right) d t \leq \inf _{r} \int_{0}^{2 \pi} \log \left(1-\left|\phi\left(r e^{i t}\right)\right|^{2}\right) d t .
$$

A direct application of Fatou's lemma gives

$$
-\int_{0}^{2 \pi} \log \left(1-\left|\phi\left(e^{i t}\right)\right|^{2}\right) d t \leq \varliminf_{r \rightarrow 1}-\int_{0}^{2 \pi} \log \left(1-\left|\phi\left(r e^{i t}\right)\right|^{2}\right) d t .
$$

As we have seen in the preceding argument, the expression on the right-hand side of (15) is equal to $-\inf _{r} \int_{0}^{2 \pi} \log \left(1-\left|\phi\left(r e^{i t}\right)\right|^{2}\right) d t$ and so we have

$$
\inf _{r} \int_{0}^{2 \pi} \log \left(1-\left|\phi\left(r e^{i t}\right)\right|^{2}\right) d t \leq \int_{0}^{2 \pi} \log \left(1-\left|\phi\left(e^{i t}\right)\right|^{2}\right) d t .
$$

It follows from (14) and (16) that (10) holds in this case.

To complete the proof of the theorem we may assume without loss of generality that $F \in S$. Now suppose $f=F \circ \phi \in E s(F)$. Then by Theorem $1, \phi \in E B_{0}$. Since $F^{\prime}(z) \neq 0$ in $\Delta$, we have $\log \left|F^{\prime}(z)\right|$ and $\log \left|F^{\prime}(\phi(z))\right|$ harmonic in $\Delta$. It follows that

$$
\int_{0}^{2 \pi} \log \left|F^{\prime}\left(\phi\left(r e^{i t}\right)\right)\right| d t=2 \pi \log \left|F^{\prime}(\phi(0))\right|=2 \pi \log \left|F^{\prime}(0)\right|=0
$$

since $F^{\prime}(0)=1$. It follows from this fact and (5) that

$$
\int_{0}^{2 \pi} \log \lambda\left(F\left(\phi\left(r e^{i t}\right)\right), \partial D\right) d t \leq \int_{0}^{2 \pi} \log \left(1-\left|\phi\left(r e^{i t}\right)\right|^{2}\right) d t .
$$

It follows from (10), (17) and the fact that $\int_{0}^{2 \pi} \log \left(1-\left|\phi\left(e^{i t}\right)\right|^{2}\right) d t=-\infty$ when $\phi \in E B_{0}$ that (9) holds and the proof is complete.

The next two theorems are technical results needed for the proof of Theorem 6.

THEOREM 4. If $F$ is a bounded univalent function analytic in $\Delta, \phi \in B_{0}$, $\left|\phi\left(e^{i t}\right)\right|<1$ for almost all $t$, then

$$
\int_{0}^{2 \pi}\left(1-\left|\phi\left(e^{i t}\right)\right|^{2}\right)^{1 / 2}\left|F^{\prime}\left(\phi\left(e^{i t}\right) e^{i \theta}\right)\right| d t<+\infty
$$

for almost all $\theta$.

PROOF. It is easy to see that the integrand in (18) is a measurable function of $(t, \theta)$ on $[0,2 \pi] \times[0,2 \pi]$ since it is almost everywhere the limit of continuous functions on $[0,2 \pi] \times[0,2 \pi]$. Since $F$ is univalent and bounded, it follows easily from Theorem 5.2 in $[\mathbf{7}$, p. 129] that there exists a constant $B$ such that

$$
\int_{0}^{2 \pi}\left|F^{\prime}\left(r e^{i \theta}\right)\right| d \theta \leq \frac{B}{(1-r)^{1 / 2-1 / 320}}
$$


for all $r<1$. Since $\left|\phi\left(e^{i t}\right)\right|<1$ for almost all $t$, (19) implies

$$
\int_{0}^{2 \pi}\left|F^{\prime}\left(\phi\left(e^{i t}\right) e^{i \theta}\right)\right| d \theta \leq \frac{B}{\left(1-\left|\phi\left(e^{i t}\right)\right|\right)^{1 / 2-1 / 320}}
$$

for almost all $t$. It follows from (20) that

$$
\int_{0}^{2 \pi}\left(1-\left|\phi\left(e^{i t}\right)\right|^{2}\right)^{1 / 2}\left|F^{\prime}\left(\phi\left(e^{i t}\right) e^{i \theta}\right)\right| d \theta \leq B
$$

for almost all $t$. Hence (21) implies that

$$
0 \leq \int_{0}^{2 \pi}\left(\int_{0}^{2 \pi}\left(1-\left|\phi\left(e^{i t}\right)\right|^{2}\right)^{1 / 2}\left|F^{\prime}\left(\phi\left(e^{i t}\right) e^{i \theta}\right)\right| d \theta\right) d t<+\infty .
$$

The Tonelli-Hobson theorem and (22) imply that

$$
0 \leq \int_{0}^{2 \pi}\left(\int_{0}^{2 \pi}\left(1-\left|\phi\left(e^{i t}\right)\right|^{2}\right)^{1 / 2}\left|F^{\prime}\left(\phi\left(e^{i t}\right) e^{i \theta}\right)\right| d t\right) d \theta<+\infty .
$$

It follows from (23) that (18) holds and the proof is complete.

REMARK. The set of $\theta$ of measure $2 \pi$ on which (18) holds depends on $\phi$. Also the exponent $1 / 2$ in (18) is not the smallest possible. Clearly $1 / 2-1 / 320$ will also suffice.

THEOREM 5. If $F$ is a univalent function analytic in $\Delta, \phi \in B_{0}$, and $\left|\phi\left(e^{i t}\right)\right|<$ 1 for almost all $t$, then

$$
\int_{0}^{2 \pi} \log \left[\left(1-\left|\phi\left(e^{i t}\right)\right|^{2}\right)^{1 / 2}\left|F^{\prime}\left(\phi\left(e^{i t}\right) e^{i \theta}\right)\right|\right] d t<+\infty
$$

for almost all $\theta$.

ProOF. We first show that it is sufficient to consider the case $F$ is univalent and bounded. If $F$ is not bounded, then $F(\Delta)$ cannot be the entire plane, so there is a point $b$ in the complement of $F(\Delta)$. By a simple argument [8, pp. 302-303] there is a number $c$ such that $g(z)=\left[(F(z)-b)^{1 / 2}+c\right]^{-1}$ is univalent and bounded in $\Delta$. So $F(z)=b+(1 / g(z)-c)^{2}$ for $|z|<1$ and $g$ is a bounded univalent function. It follows that we have

$$
\begin{aligned}
\int_{0}^{2 \pi} \log \left[\left(1-\left|\phi\left(e^{i t}\right)\right|^{2}\right)^{1 / 2}\left|F^{\prime}\left(\phi\left(e^{i t}\right) e^{i \theta}\right)\right|\right] d t \\
=2 \pi \log 2+\int_{0}^{2 \pi} \log \left|1-\operatorname{cg}\left(\phi\left(e^{i t}\right) e^{i \theta}\right)\right| d t-3 \int_{0}^{2 \pi} \log \left|g\left(\phi\left(e^{i t}\right) e^{i \theta}\right)\right| d t \\
\quad+\int_{0}^{2 \pi} \log \left(1-\left|\phi\left(e^{i t}\right)\right|^{2}\right)^{1 / 2}\left|g^{\prime}\left(\phi\left(e^{i t}\right) e^{i \theta}\right)\right| d t .
\end{aligned}
$$

Since $1-c g(z)$ and $g(z)$ are in $H^{p}$ for all $p<1 / 2[3$, p. 50], the first and second integrals on the right-hand side of $(25)$ are finite [3, p. 17]. Since we will prove that the third integral on the right is finite, the proof will be complete. that

Now suppose $g$ is a bounded univalent function analytic in $\Delta$. Theorem 4 implies

$$
\int_{0}^{2 \pi}\left(1-\left|\phi\left(e^{i t}\right)\right|^{2}\right)^{1 / 2}\left|g^{\prime}\left(\phi\left(e^{i t}\right) e^{i \theta}\right)\right| d t<+\infty
$$


for almost all $\theta$. It is easy to deduce (24) from this fact and this completes the proof.

REMARK. The set of $\theta$ of measure $2 \pi$ on which (24) holds depends on $\phi$.

THEOREM 6. If $F$ is a univalent function analytic in $\Delta, \phi \in E B_{0}$, and $\left|\phi\left(e^{i t}\right)\right|$ $<1$ for almost all $t$, then

$$
\int_{0}^{2 \pi} \log \lambda\left(F\left(\phi\left(e^{i t}\right) e^{i \theta}\right), \partial D\right) d t=-\infty
$$

for almost all $\theta$.

ProOF. Since $F$ is univalent we have from (5) that

$$
\lambda\left(F\left(\phi\left(e^{i t}\right) e^{i \theta}\right), \partial D\right) \leq\left(1-\left|\phi\left(e^{i t}\right)\right|^{2}\right)\left|F^{\prime}\left(\phi\left(e^{i t}\right) e^{i \theta}\right)\right|
$$

for almost all $t$ and all $\theta$. It follows from (27) that

(28)

$$
\begin{aligned}
& \int_{0}^{2 \pi} \log \lambda\left(F\left(\phi\left(e^{i t}\right) e^{i \theta}\right), \partial D\right) d t \\
& \quad \leq \frac{1}{2} \int_{0}^{2 \pi} \log \left(1-\left|\phi\left(e^{i t}\right)\right|^{2}\right) d t+\int_{0}^{2 \pi} \log \left(1-\left|\phi\left(e^{i t}\right)\right|^{2}\right)^{1 / 2}\left|F^{\prime}\left(\phi\left(e^{i t}\right) e^{i \theta}\right)\right| d t .
\end{aligned}
$$

Since $\phi \in E B$, we have $\int_{0}^{2 \pi} \log \left(1-\left|\phi\left(e^{i t}\right)\right|^{2}\right) d t=-\infty[3$, p. 125] and so (26) follows from this fact, (24), and (28).

REMARK. The set of $\theta$ of measure $2 \pi$ on which (26) holds depends on $\phi$.

COROLlaRY. If $F$ is a univalent function analytic in $\Delta, \phi \in B_{0}$, and $F \circ \phi \in$ $E s(F)$, then

$$
\int_{0}^{2 \pi} \log \lambda\left(F\left(\phi\left(e^{i t}\right) e^{i \theta}\right), \partial D\right) d t=-\infty
$$

for almost all $\theta$.

PROOF. This follows directly from Theorem 1 and Theorem 6 .

Our next result generalizes the previous theorem.

THEOREM 7. If $F$ is a univalent function analytic in $\Delta, \phi \in E B_{0},\left|\phi\left(e^{i t}\right)\right|<1$ for almost all $t$, and $\psi$ is an inner function such that $|\psi(0)| \neq 1$, then

$$
\int_{0}^{2 \pi} \log \lambda\left(F\left(\phi\left(e^{i t}\right) \psi\left(e^{i \theta}\right)\right), \partial D\right) d t=-\infty
$$

for almost all $\theta$.

PROOF. We just prove that under the assumption that $F$ is a bounded univalent function in $\Delta$ then

$$
\int_{0}^{2 \pi}\left(1-\left|\phi\left(e^{i t}\right)\right|^{2}\right)^{1 / 2}\left|F^{\prime}\left(\phi\left(e^{i t}\right) \psi\left(e^{i \theta}\right)\right)\right| d t<+\infty
$$

for almost all $\theta$. The proof of (30) follows from (31) in the way that Theorem 6 was deduced from Theorems 4 and 5 . We note that Fatou's lemma gives

$$
\begin{aligned}
\int_{0}^{2 \pi} & \left(1-\left|\phi\left(e^{i t}\right)\right|^{2}\right)^{1 / 2}\left|F^{\prime}\left(\phi\left(e^{i t}\right) \psi\left(e^{i \theta}\right)\right)\right| d \theta \\
& \leq \varliminf_{r \rightarrow 1} \int_{0}^{2 \pi}\left(1-\left|\phi\left(e^{i t}\right)\right|^{2}\right)^{1 / 2}\left|F^{\prime}\left(\phi\left(e^{i t}\right) \psi\left(r e^{i \theta}\right)\right)\right| d \theta
\end{aligned}
$$


Littlewood's inequality $[3$, pp. 10-11; 6, p. 243] can easily be generalized so that we have

$$
\int_{0}^{2 \pi}\left|F^{\prime}\left(\phi\left(e^{i t}\right) \psi\left(r e^{i \theta}\right)\right)\right| d \theta \leq \frac{r+|\psi(0)|}{r-|\psi(0)|} \int_{0}^{2 \pi}\left|F^{\prime}\left(\phi\left(e^{i t}\right) r e^{i \theta}\right)\right| d \theta
$$

whenever $|\psi(0)|<r$. Since $F$ is a bounded univalent function, (19) and (13) imply that when $|\psi(0)|<r$

$$
\int_{0}^{2 \pi}\left|F^{\prime}\left(\phi\left(e^{i t}\right) \psi\left(r e^{i \theta}\right)\right)\right| d \theta \leq \frac{r+|\psi(0)|}{r-|\psi(0)|} \frac{B}{\left(1-\left|\phi\left(e^{i t}\right)\right| r\right)^{1 / 2-1 / 320}} .
$$

It follows from (32) and (34) that we have

$$
\int_{0}^{2 \pi}\left(1-\left|\phi\left(e^{i t}\right)\right|^{2}\right)^{1 / 2}\left|F^{\prime}\left(\phi\left(e^{i t}\right) \psi\left(e^{i \theta}\right)\right)\right| d \theta \leq \frac{1+|\psi(0)|}{1-|\psi(0)|} B .
$$

We infer from (35) that

$$
\int_{0}^{2 \pi}\left(\int_{0}^{2 \pi}\left(1-\left|\phi\left(e^{i t}\right)\right|^{2}\right)^{1 / 2}\left|F^{\prime}\left(\phi\left(e^{i t}\right) \psi\left(e^{i \theta}\right)\right)\right| d \theta\right) d t<+\infty
$$

Hence (31) follows from (36) and the Tonelli-Hobson theorem.

REMARK. The set of $\theta$ of measure $2 \pi$ on which (30) holds depends on $\phi$ and $\psi$.

\section{REFERENCES}

1. Y. Abu-Muhanna, On extreme points of subordination families, Proc. Amer. Math. Soc. 87 (1983), 439-443.

2. L. Brickman, T. H. MacGregor, and D. R. Wilken, Convex hulls of some classical families of univalent functions, Trans. Amer. Math. Soc. 156 (1971), 91-107.

3. P. L. Duren, Theory of $H^{p}$ spaces, Academic Press, New York, 1970.

4. D. J. Hallenbeck, Extreme points of subordination families with univalent majorants, Proc. Amer. Math. Soc. 91 (1984), 54-58.

5. D. J. Hallenbeck and T. H. MacGregor, Linear problems and convexity techniques in geometric function theory, Monographs and Studies in Mathematics 22, Pitman, London, 1984.

6. E. Hille, Analytic function theory, Vol. II, Chelsea, New York, 1962.

7. Chr. Pommerenke, Univalent functions, Vandenhoeck and Ruprecht, Göttingen, 1975.

8. W. Rudin, Real and complex analysis, 3rd ed., McGraw-Hill, New York, 1987.

Department of Mathematical Sciences, University of Petroleum and MinERALS, DhaHRAN, SaUdi ARABIa

Department of Mathematical Sciences, University of Delaware, Newark, DELAWARE 19716 